Check for updates

Cite this: Mater. Horiz., 2019, 6,1283

DOI: 10.1039/c9mh90031d

rsc.li/materials-horizons

\section{Correction: A siRNA-induced peptide co-assembly system as a peptide-based siRNA nanocarrier for cancer therapy}

\author{
Wenjun $\mathrm{Li}^{\mathrm{a}}{ }^{2}$ Dongyuan Wang, ${ }^{\mathrm{a}}$ Xiaodong Shi, ${ }^{a}$ Jingxu $\mathrm{Li}{ }^{\mathrm{a}}$ Yue $\mathrm{Ma},{ }^{a}$ Yanding Wang, ${ }^{a}$ \\ Tingting $\mathrm{Li}^{\mathrm{b}}$, Jianing Zhang, ${ }^{a}$ Rongtong Zhao, ${ }^{a}$ Zhiqiang $\mathrm{Yu}^{,}{ }^{\mathrm{C}}$ Feng $\mathrm{Yin}^{{ }^{* a}}$ and \\ Zigang Li*a
}

Correction for 'A siRNA-induced peptide co-assembly system as a peptide-based siRNA nanocarrier for cancer therapy' by Wenjun Li et al., Mater. Horiz., 2018, 5, 745-752.

The authors regret an error in Fig. 3E in the originally published manuscript. The correct version of Fig. 3 is shown below.

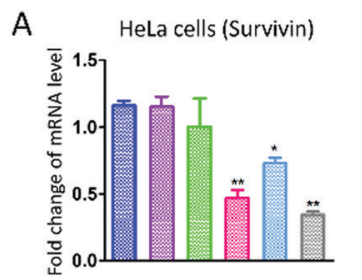

E

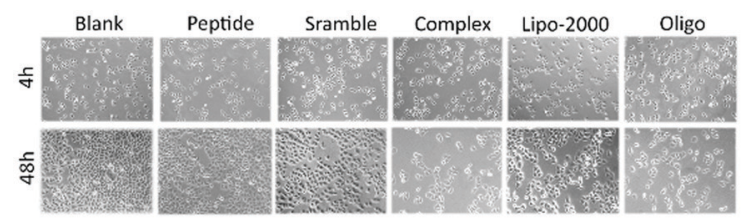

C

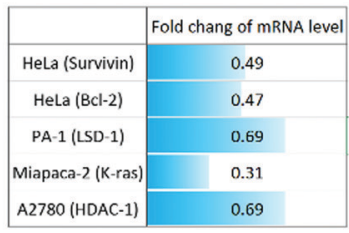

$\mathrm{F}$

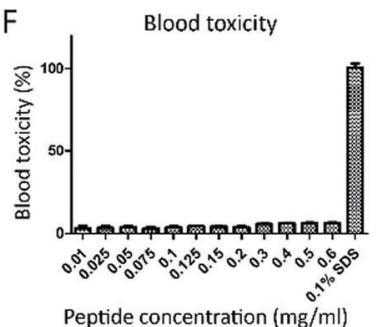

D

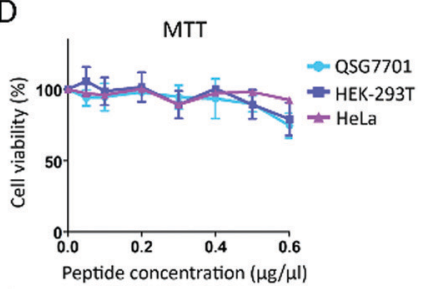

G

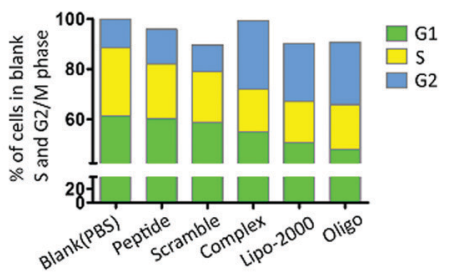

Fig. 3 Cell assay evaluation of the peptide-siRNA co-assembled nanoparticles. Scramble: peptide-siRNA (Scramble) complex. (A) RT-PCR analysis of HeLa cells transfected with peptide-siRNA (Survivin) co-assembled nanoparticles. (B) RT-PCR analysis of HeLa cells transfected with peptide-siRNA (Bcl-2) co-assembled nanoparticles. Both the delivery of Survivin and Bcl-2 siRNA showed a significant knockdown in HeLa cells. ${ }^{*}<0.05,{ }^{\star *} P<0.01$ vs. PBS (blank). (C) The summary of the fold change of the mRNA level in different cell lines by RT-PCR analysis (HeLa cells, PA-1 cells, Miapaca-2 cells and A2780 cells), transfected with different peptide-siRNA nanoparticles. The detailed RT-PCR results are provided in Fig. S12 (ESI $†$ ). (D) MTT assay of peptide Wpc in Immortalized cells (HEK-293T cells), normal liver cells (QSG7701 cells) and tumor cells (HeLa cells). (E) Inverted microscope image of HeLa cells treated with peptide siRNA co-assembled nanoparticles after $4 \mathrm{~h}$ and $48 \mathrm{~h}$. (F) Blood cytotoxicity assay of peptide Wpc in fresh mouse blood with $0.1 \%$ SDS as a positive control. (G) Percentage of HeLa cells in each mitotic phase after being treated with different samples. HeLa cells were transfected with (2.0 mg siRNA with 50 mg peptide) nanoparticles for $48 \mathrm{~h}$. Then they were stained and analyzed by flow cytomerty. A clearly higher proportion of HeLa cells were in the G2 phase after being treated with peptide-siRNA nanoparticles or positive controls than any other treatments. All the experiments were conducted twice with consistent results. Error bars represent SD of the data. The statistical differences between the control group and experimental groups were analyzed by one-way ANOVA.

The Royal Society of Chemistry apologises for these errors and any consequent inconvenience to authors and readers.

\footnotetext{
${ }^{a}$ State Key Laboratory of Chemical Oncogenomics, School of Chemical Biology and Biotechnology, Peking University Shenzhen Graduate School, Shenzhen, 518055, China. E-mail: lizg@pkusz.edu.cn; Fax:+86-755-2603-3174; Tel: +86-755-2603-3616

${ }^{b}$ School of Advanced Material, Peking University Shenzhen Graduate School, Shenzhen, 518055, China

${ }^{c}$ School of Pharmaceutical Sciences, Guangdong Provincial Key Laboratory of New Drug Screening, Southern Medical University, Guangzhou 510515, China
} 